\title{
New Design of Compressor Blade with Moderate Material Properties for Performance Optimization
}

\author{
Yuchao Song, ${ }^{1}$ Hongliang Yu, ${ }^{2}$ Jianbin Liao, ${ }^{2}$ \\ Chih-Cheng Chen, ${ }^{3,4 *}$ Chaoming Huang, ${ }^{2 * *}$ and Christopher Chun Ki Chan ${ }^{5}$ \\ ${ }^{1}$ Marine Engineering College, Dalian Maritime University, 1 Linghai Road, Dalian 116026, China \\ ${ }^{2}$ Marine Engineering College, Jimei University, 185 Yinjiang Road, Jimei District, Xiamen 361021, China \\ ${ }^{3}$ Department of Automatic Control Engineering, Feng Chia University 40724, Taiwan \\ ${ }^{4}$ Department of Aeronautical Engineering, Chaoyang University of Technology, Taichung 413310, Taiwan \\ ${ }^{5}$ Department of Information Management, Chaoyang University of Technology, Taichung 413310, Taiwan
}

(Received October 21, 2020; accepted April 5, 2021)

Keywords: centrifugal compressor wheel, blade, leading-edge design, pressure ratio, efficiency

When optimizing the performance of a high-speed centrifugal compressor, the mechanical properties of the blade material must be suitable for the sensor mounted on the compressor. We designed a new blade with a leading edge having a multistep concave-convex profile, inspired by research on bionics and BladeGen software (an interactive turbomachinery design tool). We compared its performance with that of an original blade having a straight-line leading edge. For the new blade, TurboGrid refinement technology is used to ensure the meshing of the profile's structure. Computation results show that the deformation displacement of the new blade is reduced by $4.26 \%$ and that it has better mechanical properties. Moreover, the static pressure ratio and the total pressure ratio of the new wheel are both higher than those of the original wheel over a wide range of mass flow rates. The efficiency of the new wheel decreases slightly by about $1 \%$ at an intermediate mass flow rate, but the total-total efficiency and total-static efficiency of the new wheel are increased by $0.35 \%$ and $6.36 \%$ at a high mass flow rate of $0.1781 \mathrm{~kg} / \mathrm{s}$ and by $0.02 \%$ and $0.84 \%$ at a low mass flow rate of $0.0832 \mathrm{~kg} / \mathrm{s}$, respectively. Thus, the new blade design improves the mechanical properties of the blade, and the smaller stress is beneficial for compressor performance optimization during the sensing process.

\section{Introduction}

As the critical component of a turbocharger, a centrifugal compressor is widely used in vehicles, ships, and aviation. Its structure is small-scale, but the rotating speed is higher than 10,000 revolutions per minute ( $\mathrm{rpm}$ ), and the blade is subjected to severe transonic aerodynamical conditions, which may affect performance and sensor reliability. Sensors are used in a compressor for performance measurement and blade failure detection. Severe compressor vibration can cause failures and maintenance issues for sensors. Thus, the compressor performance and sensor working conditions should be clearly determined during the design

\footnotetext{
*Corresponding author: e-mail: ccc@gm.cyut.edu.tw

${ }^{* *}$ Corresponding author: e-mail: hchaoming@163.com

https://doi.org/10.18494/SAM.2021.3178
} 
stage. The principles and technology for improving compressor wheel performance have become hot topics in industry, with the aim of achieving better material properties. Scholars worldwide have carried out in-depth research on the wheel shape, blade leading edge, trailing edge ellipse parameters, and tip clearance for high-performance centrifugal compressors for a long time. ${ }^{(1-6)}$ Following aerodynamic optimization design, structural reliability and sensing technology are generally analyzed by the finite element method. ${ }^{(7,8)}$

Meanwhile, with the increasing demand for energy-saving technology globally, optimizing material reliability and improving performance are a focus of research institutes and enterprises. The internal structure and aerodynamic conditions of a compressor are very complex. A professional procedure called forward design is used to model wheel blades, then computational fluid dynamics (CFD) technology is used to simulate and compare the performance of compressors. Given the interactive development of science, technology, and interdependent disciplines, bionic technology has become a vibrant new field that has attracted the interest of many researchers. A fish-shaped blade design with a microscale surface and bionic structure is one example of a design that has improved compressor performances,${ }^{(9-12)}$ resulting in good material properties. Some scholars have studied the material surface processing technology for compressor blades. ${ }^{(13)}$

Furthermore, compressor sensors are usually mounted in holes opened on the compressor shroud and pipes for direct measurement. The gap between the probe and the rotating wheel is minimal. Optimal performance, favorable material properties, and pristine sensor operation are essential elements of a turbocharger and engine system. All these elements should be considered in the development of high-performance compressors.

In this paper, we propose a new blade design with improved performance and materials. We designed a blade with a bionic concave-convex leading edge and studied its material properties and performance to optimize a prototype eight-blade compressor wheel. The leading edge of the new blade is from the hub to the shroud. The new bionic wheel is modeled and calculated using ANSYS CFX software, and the material properties and aerodynamic parameters are compared between the new compressor and the original compressor simultaneously. The bionic blade is of great significance to improve the overall performance of high-speed centrifugal compressors with optimal material properties and sensor operation.

\section{Mechanics and Flow Formula}

For a rotating wheel, the dynamic equation can be expressed as follows:

$$
[M]\{\ddot{u}\}+[C]\{\dot{u}\}+[K]\{u\}=\{F\},
$$

where $[M]$ is the mass matrix, $[C]$ is the damping matrix, $[K]$ is the stiffness matrix, $\{F\}$ is the load vector, $\{u\}$ is the wheel displacement vector, $\{\dot{u}\}$ is the wheel velocity vector, and $\{\ddot{u}\}$ is the wheel acceleration vector. 
As the blade rotates, the internal force and centrifugal force are in equilibrium:

$$
\left(k-M \omega^{2}\right) u=M r \omega^{2},
$$

where $r$ is the radius of rotation of the blade mass point. Thus, there is an actual stiffness $k^{\prime}$, whose expression is

$$
k^{\prime}=k-M \omega^{2} .
$$

According to Eq. (3), the actual stiffness is reduced at a high rotating speed. Therefore, it is important to calculate the rotating mechanics of the blade.

Owing to the effect of an external force on the structure, a stress field is also generated in the blade. This stress field generates a stress stiffness matrix, which is superimposed on the original stiffness matrix and increases (or decreases) the stiffness of the structure. This phenomenon is called stress hardening. Under the rotating inertia force of the rotating blade, such stress hardening always increases the natural frequency of the blade.

In addition to affecting the mechanics of the material, the rotation of the blade also affects the airflow in the compressor. According to the Prandtl formula, the distribution of fluid velocity depends on the wall shear stress $\tau_{w}$ of the blade, fluid density $\rho$, dynamic viscosity $\mu$, distance from the wall of the rotating blade $y$, and wall roughness $\Delta$ as follows:

$$
\vec{v}_{x}=F\left(\tau_{w}, \rho, \mu, y, \Delta\right) .
$$

In this paper, the blade surface is rough, so the wall roughness $\Delta$ is used to analyze the wheel's hydrodynamics. All the material mechanics and the flow effects are important factors in performance optimization and the sensing process.

\section{Material Profile Design of Blade Leading Edge}

A small high-speed centrifugal compressor generally has a rotation speed in the range of 70000 to $250000 \mathrm{rpm}$. There are many challenges to improving the pressure ratio and efficiency under high-speed aerodynamic conditions while ensuring the mechanical properties of the material and sensing reliability. To improve the pressure ratio and efficiency, the optimization of the blade shape is our primary aim. Bionics studies have found that the convex structure of the leading edge of the wing of a long-eared owl can affect the pressure distribution and the wake vortex on the pressure surface and suction surface. ${ }^{(14)}$ On the basis of the principle of aerodynamics, a bionic configuration is adopted for the impeller of a high-speed centrifugal compressor. Table 1 shows the dimensions of the original wheel and Fig. 1 shows a meridional section view of the designed compressor, including the inlet domain, flow passage, and vaneless diffuser domain. There are also profile control points on the leading edge. 
Table 1

Main parameters of the original wheel.

\begin{tabular}{lc}
\hline Parameter & Value \\
\hline Hub diameter at inlet & $10 \mathrm{~mm}$ \\
Shroud diameter at inlet & $35 \mathrm{~mm}$ \\
Wheel diameter at outlet & $49 \mathrm{~mm}$ \\
Diffuser diameter at outlet & $86 \mathrm{~mm}$ \\
Diffuser width at outlet & $2.3 \mathrm{~mm}$ \\
Rotation speed & $190000 \mathrm{rpm}$ \\
Mass flow rate & $0.08-0.18 \mathrm{~kg} / \mathrm{s}$ \\
\hline
\end{tabular}

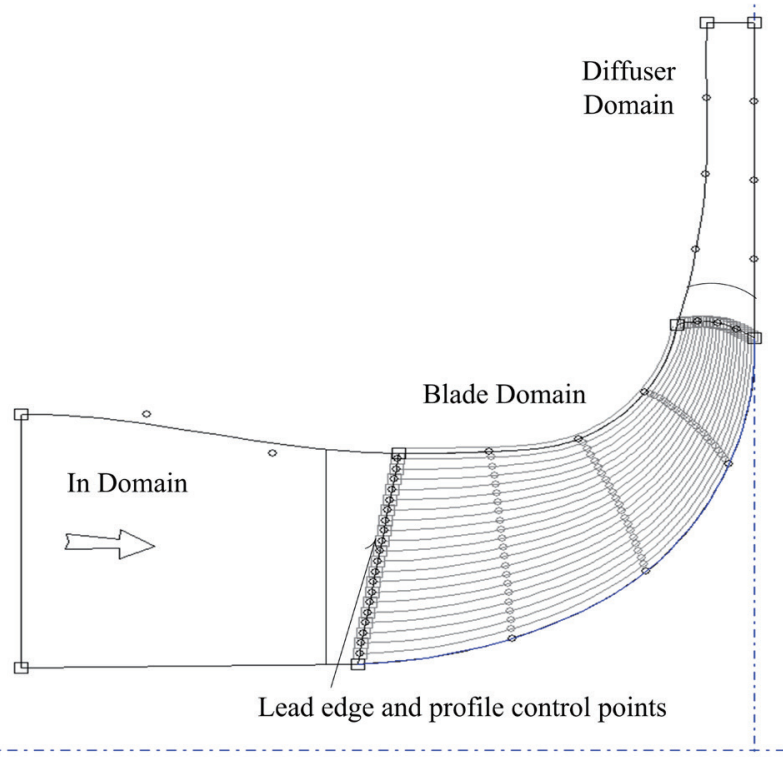

Fig. 1. Meridional section of compressor.

On the basis of the original wheel, a new leading edge with a convex and concave profile is designed using Bezier curves in BladeGen. Unlike the previous straight-line leading edge, the convexity heights of the new leading edge are set from the hub to the shroud, which are 0.01 , $0.02,0.03,0.04,0.05$, and $0.06 \mathrm{~mm}$. The chord length of the centerline of the two adjacent convexities is $1.8285 \mathrm{~mm}$. The stepped leading edge of the blade shown in Fig. 2(b) is designed according to the above values. The volume of the prototype wheel blade [see Fig. 2(a)] is $1.95 \times$ $10^{-7} \mathrm{~m}^{3}$ and the surface area is $5.61 \times 10^{-4} \mathrm{~m}^{2}$; both values were obtained by SOLIDWORKS. The volume of the bionic wheel blade is $1.93 \times 10^{-7} \mathrm{~m}^{3}$ and the surface area is $5.60 \times 10^{-4} \mathrm{~m}^{2}$. By optimization of the design, the blade volume is decreased by $1.03 \%$, the surface area is decreased by $0.18 \%$, and the area/volume ratio is increased by $0.86 \%$.

When improving and optimizing a blade design, it is necessary to ensure suitable material mechanical properties and sensor reliability to obtain optimal aerodynamic parameters. Figure 3 shows that the maximum displacements of the original and new blades are $9.84 \times 10^{-2}$ and $9.42 \times 10^{-2} \mathrm{~mm}$, respectively, at a speed of $190000 \mathrm{rpm}$, both of which are less than the tip clearance of $0.33 \mathrm{~mm}$. The deformation displacement of the new blade is reduced by $4.26 \%$, showing that it has better material mechanical conditions. These material mechanical conditions can reduce the vibration level, which is beneficial for the mounted sensors.

\section{Numerical Modeling of Wheel}

As the inlet air changes the flow direction in the passage of a centrifugal compressor, the secondary flow and flow loss will reduce the efficiency and pressure ratio of the compressor. ${ }^{(15)}$ The new step on the leading edge is parallel along the blade span direction in the flow channel. To verify the independence of the result of numerical modeling on the grid, Table 2 shows the 


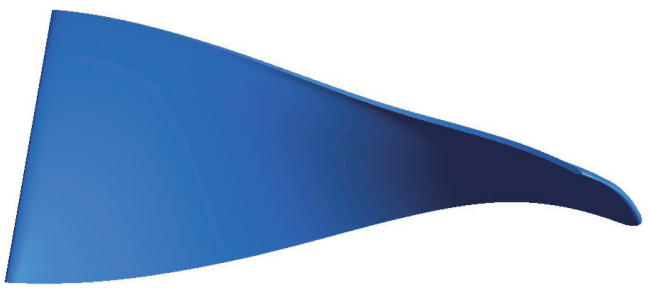

(a)

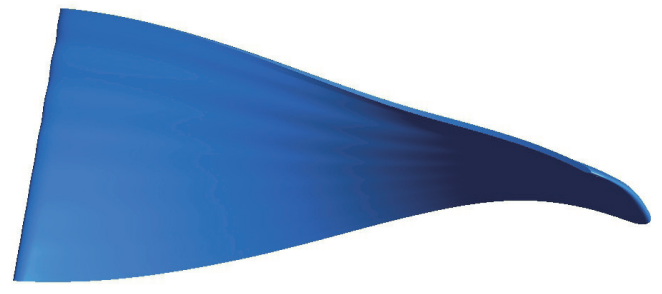

(b)

Fig. 2. (Color online) Profile of blade. (a) Original blade. (b) New blade.

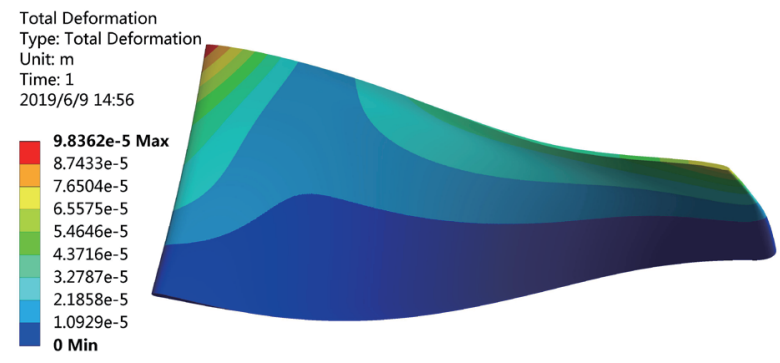

(a)

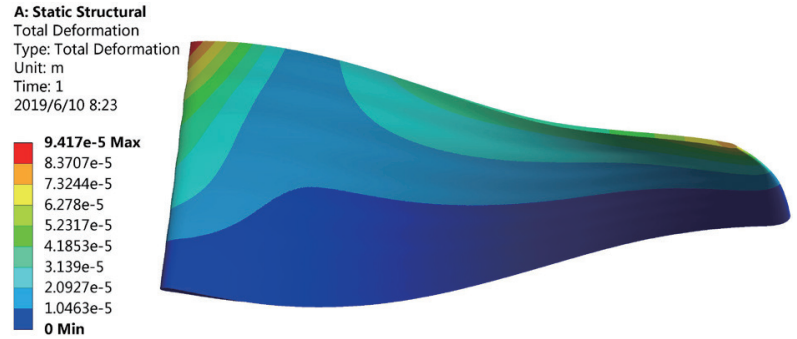

(b)

Fig. 3. (Color online) Rotating displacement contour of deformation. (a) Original blade. (b) New blade.

Table 2

Dependence of total-total efficiency on grid number.

\begin{tabular}{lcccc}
\hline Mass flow rate $(\mathrm{kg} / \mathrm{s})$ & 250000 & 600000 & 1200000 & 5500000 \\
\hline 0.0832 & 0.7156 & 0.7218 & 0.7171 & 0.7169 \\
0.1013 & 0.7363 & 0.7447 & 0.7455 & 0.7466 \\
0.1149 & 0.7584 & 0.7684 & 0.7687 & 0.7687 \\
0.1313 & 0.7926 & 0.8032 & 0.8038 & 0.8038 \\
0.1465 & 0.8176 & 0.8305 & 0.8307 & 0.8184 \\
0.1628 & 0.8300 & 0.8346 & 0.8191 & 0.8370 \\
0.1781 & 0.7950 & 0.7675 & 0.7726 & 0.7763 \\
\hline
\end{tabular}

total-total efficiency of the original wheel with different grid numbers. The efficiency fluctuates slightly among the grid numbers of 250000, 600000, 1200000, and 5500000. Furthermore, the distance of the first mesh cell off the nearest wall should be suitable to ensure the accurate simulation of the flow field. The non-dimensional wall distance is denoted $\mathrm{Y}+$. Since the $\mathrm{Y}+$ value on the rotating blade's surface is generally required to be less than 2 during the numerical computation, the mesh with a grid number of 5500000 meets this requirement. Figure 4 shows the leading-edge grid used in TurboGrid, which describes the stepped characteristic of the blade leading edge. As shown in Fig. 5, the Y+ value of the blade wall is below 1. Considering the parallel numerical computation, a grid number of 5500000 is selected for modeling. 


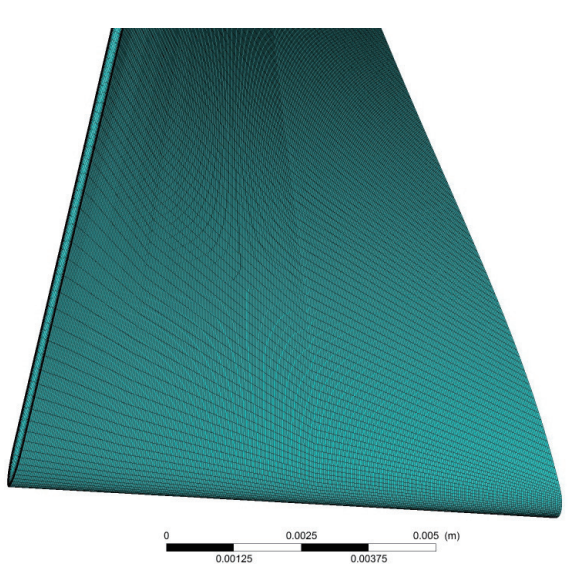

(a)

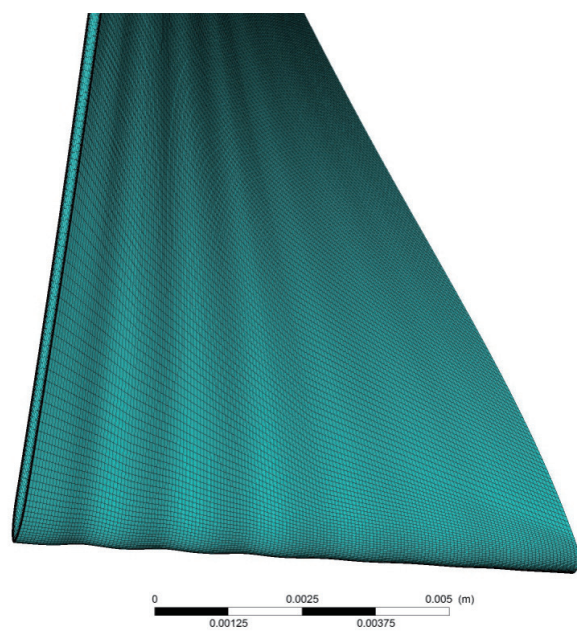

(b)

Fig. 4. (Color online) Grid on each blade with 5500000 elements. (a) Original blade. (b) New blade.



(a)

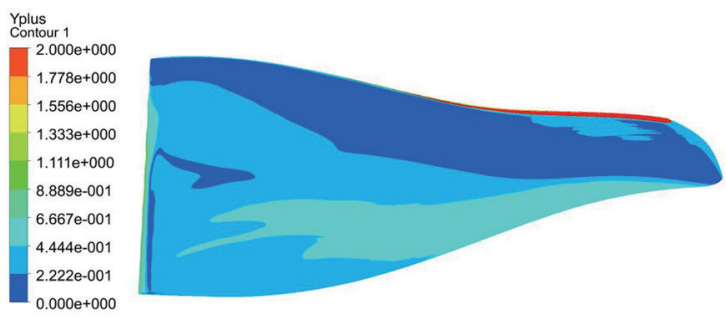

(b)

Fig. 5. (Color online) Y+ distribution of rotating blade with 5500000 elements. (a) Original blade. (b) New blade.

To evaluate compressor aerodynamics, the computation domain is usually divided into the stationary domain and rotating domain according to the rotating condition. In terms of boundary settings, the inlet region and outlet region of the vaneless diffuser are static regions, all the walls are set as static nonsliding surfaces, the flow passage domain is set as the rotating region, the walls of the blade and hub are rotating walls, and the wall of the shroud is a static nonsliding wall. The gas (air) is an ideal continuous fluid. A total energy heat transfer model (SST Turbulence Model) and a high-speed compressible wall heat transfer model considering the viscosity factor are adopted. As shown in Fig. 6, the corresponding periodic rotating walls and internal domain mixing interfaces need to be established on both sides of the single passage in the numerical computation. A multicore large-memory parallel mode is used to solve the flow problem. 


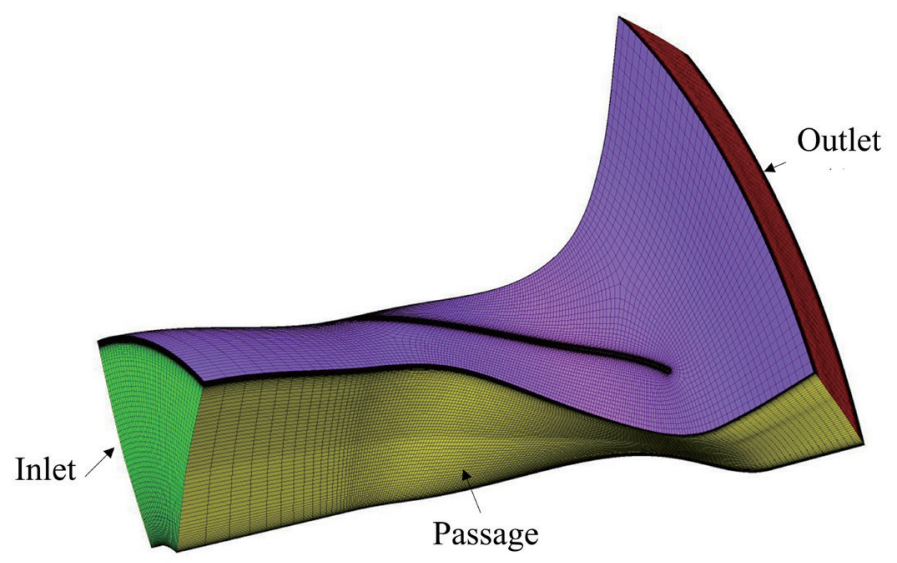

Fig. 6. (Color online) Grid of single passage used in computation.

\section{Performance and Discussion}

\subsection{Performance results}

After obtaining the convergence criterion, the TurboGrid module is used to calculate and analyze the compressor performance parameters based on CFX-Post codes. The compressor pressure ratio $\pi$ and efficiency $\eta$ are defined as follows.

Static pressure ratio: $\pi_{\text {static }}=P_{\text {Out }} / P_{\text {In }}$

Total pressure ratio: $\pi_{\text {total }}=P_{\text {TotalOut }} / P_{\text {Totalln }}$

Total-static efficiency: $\eta_{\text {total-static }}=\left(1-P_{\text {Out }} / P_{\text {Totaln }}\right)^{(\gamma-1) / \gamma} /\left(1-t_{\text {TotalOut }} / t_{\text {Totalln }}\right)$

Total-total efficiency: $\eta_{\text {total-total }}=\left(1-P_{\text {TotalOut }} / P_{\text {TotalIn }}\right)^{(\gamma-1) / \gamma} /\left(1-t_{\text {TotalOut }} / t_{\text {TotalIn }}\right)$

Here $P_{\text {In }}, P_{\text {TotalIn }}, P_{\text {Out }}$, and $P_{\text {TotalOut }}$ are the static pressure and total pressure at the inlet and outlet of the passage, respectively, $t_{\text {TotalIn }}$ is total temperature of the inlet, and $t_{\text {TotalOut }}$ is the total temperature of the outlet.

Figure 7 shows the operating characteristics of the original compressor at $190000 \mathrm{rpm}$. It can be seen from the pressure ratio curve that the pressure ratio fluctuates as a function of the mass flow rate. The total-total efficiency and total-static efficiency first increase and then decrease. The parameter change ratio is defined as the difference between the values for the new wheel and the original wheel. The variation ratio of the four wheel performance parameters is shown in Fig. 8. The static pressure ratio and total pressure ratio increased in the entire mass flow rate range, except at intermediate mass flow rates of 0.1013 and $0.1628 \mathrm{~kg} / \mathrm{s}$, where they decreased slightly by $0.5 \%$. From 0.1013 to $0.1313 \mathrm{~kg} / \mathrm{s}$, the efficiency decreases by about $1 \%$. The pressure ratio decreases at intermediate mass flow rates and then increases for the original wheel. When the mass flow rate is $0.0832 \mathrm{~kg} / \mathrm{s}$, the static pressure ratio and total pressure ratio are increased by $0.61 \%$ and $0.07 \%$ and the total-static efficiency and total-total efficiency are increased by $0.02 \%$ and $0.84 \%$, respectively. When the mass flow rate is $0.1781 \mathrm{~kg} / \mathrm{s}$, the static pressure ratio 


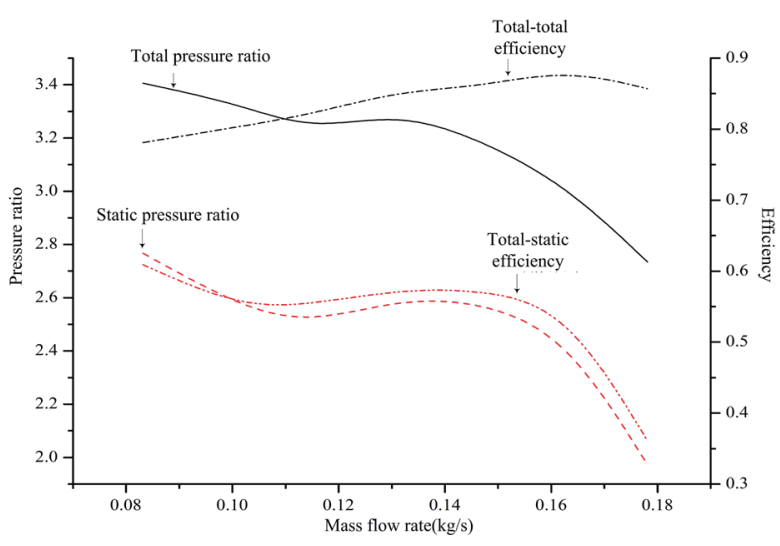

Fig. 7. (Color online) Performance of original wheel at $190000 \mathrm{rpm}$.

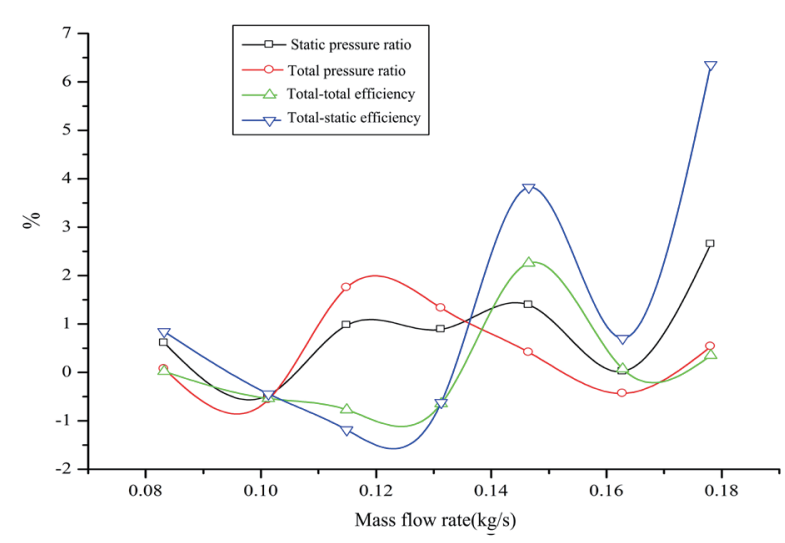

Fig. 8. (Color online) Comparison of pressure ratio between new and original wheels.

and total pressure ratio are increased by $2.65 \%$ and $0.54 \%$ and the total-static efficiency and total-total efficiency are increased by $6.36 \%$ and $0.35 \%$, respectively. These results have practical engineering significance for further optimization of the compressor.

\subsection{Discussion of characteristics}

When the wheel is rotating at a high speed, the field characteristics characterize its performance parameters. Figures 9-11 show the distribution of entropy increase in the passage at a high mass flow rate and low mass flow rate when the blade height is 0.1, 0.5, and 0.9, respectively. According to Fig. 10, when the blade height is 0.5 , the entropy increases over a large area when the mass flow rate is $0.0832 \mathrm{~kg} / \mathrm{s}$, and the maximum entropy occurs on the suction surface side of the blade leading edge and on the suction surface at the rear of the passage. The entropy of the original wheel value is $261.2 \mathrm{~J} / \mathrm{kg} / \mathrm{k}$, while the maximum entropy of the new wheel is $258.0 \mathrm{~J} / \mathrm{kg} / \mathrm{K}$, a decrease of about $1.23 \%$. In the high-entropy region with entropy higher than $220 \mathrm{~J} / \mathrm{kg} / \mathrm{K}$, the new impeller area in Fig. 10(c) is significantly smaller than that in Fig. 10(a). When the mass flow rate is $0.1781 \mathrm{~kg} / \mathrm{s}$, as shown in Figs. 10(b) and 10(d), the entropy is high in the outlet and on the trailing-edge suction side. The maximum entropy of the new wheel is $500 \mathrm{~J} / \mathrm{kg} / \mathrm{K}, 2.15 \%$ less than that of the original wheel of $511 \mathrm{~J} / \mathrm{kg} / \mathrm{K}$.

In Fig. 9, the same phenomenon can also be seen in the distribution of channel entropy for the low blade height of 0.1. In the new impeller, the high-entropy area is reduced, especially at the outlet of the blade trailing edge. When the blade height is 0.9 (Fig. 11), the entropy is greatest. When the mass flow rate is $0.0832 \mathrm{~kg} / \mathrm{s}$, the high-entropy area of the new wheel is smaller than that of the original wheel. When the mass flow rate is increased to $0.1781 \mathrm{~kg} / \mathrm{s}$, the entropy at the outlet of the trailing edge of the new wheel is better than that of the original wheel. 


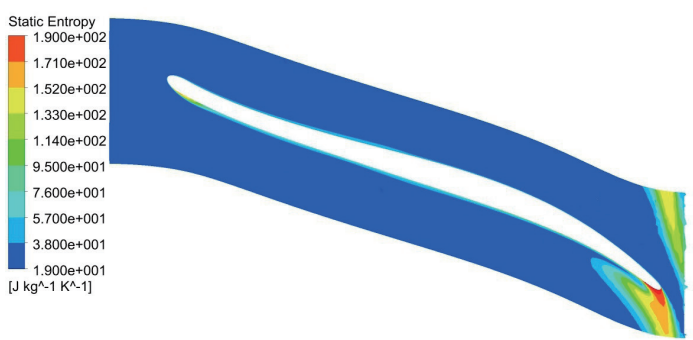

(a)

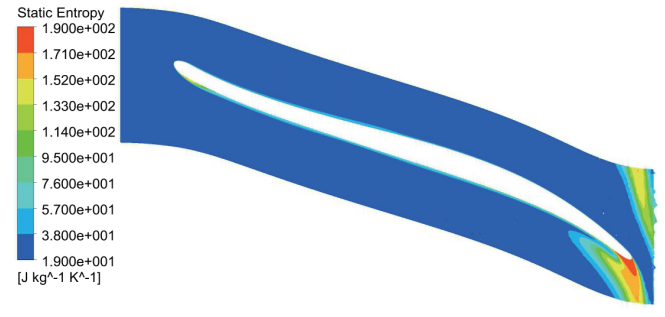

(c)

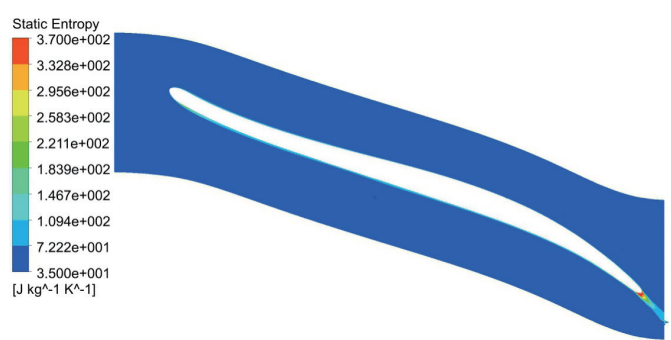

(b)

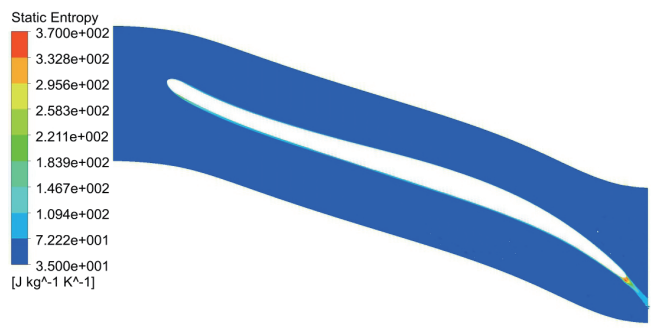

(d)

Fig. 9. (Color online) Static entropy of blade-to-blade section at blade height of 0.1. (a) Original blade at 0.0832 $\mathrm{kg} / \mathrm{s}$. (b) Original blade at $0.1781 \mathrm{~kg} / \mathrm{s}$. (c) New blade at $0.0832 \mathrm{~kg} / \mathrm{s}$. (d) New blade at $0.1781 \mathrm{~kg} / \mathrm{s}$.

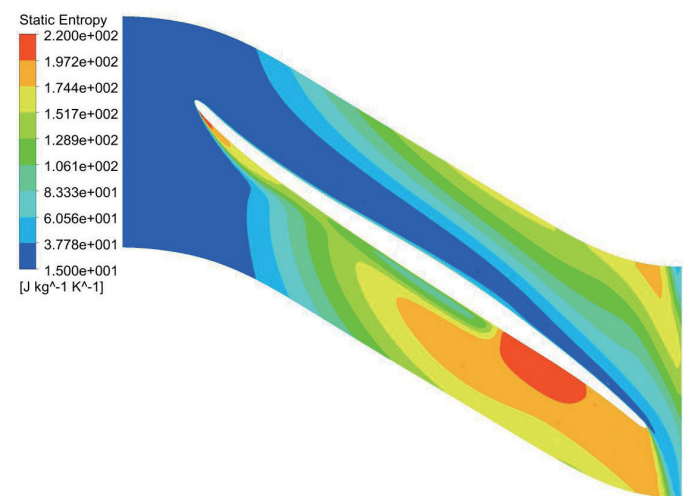

(a)

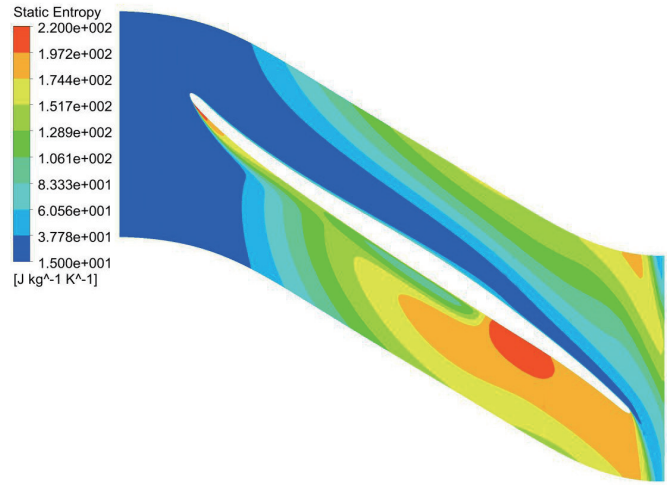

(c)

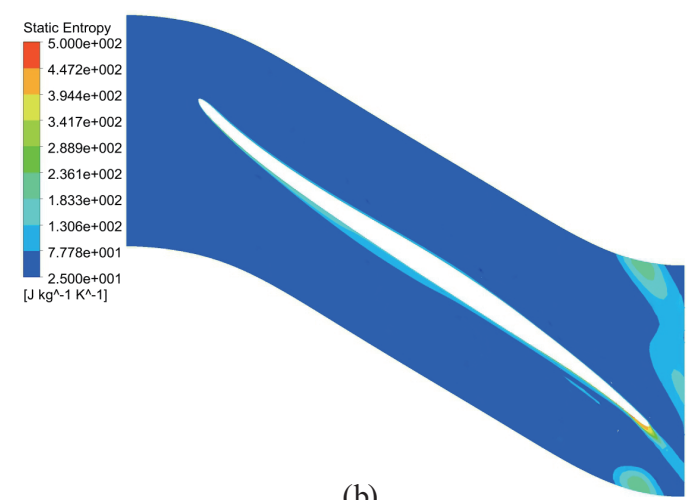

(b)



(d)

Fig. 10. (Color online) Static entropy of blade-to-blade section at blade height of 0.5 . (a) Original blade at 0.0832 $\mathrm{kg} / \mathrm{s}$. (b) Original blade at $0.1781 \mathrm{~kg} / \mathrm{s}$. (c) New blade at $0.0832 \mathrm{~kg} / \mathrm{s}$. (d) New blade at $0.1781 \mathrm{~kg} / \mathrm{s}$. 


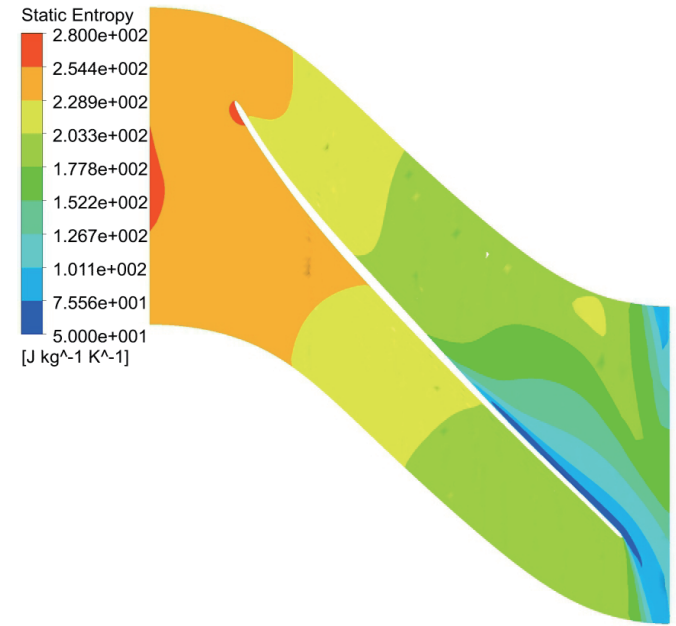

(a)

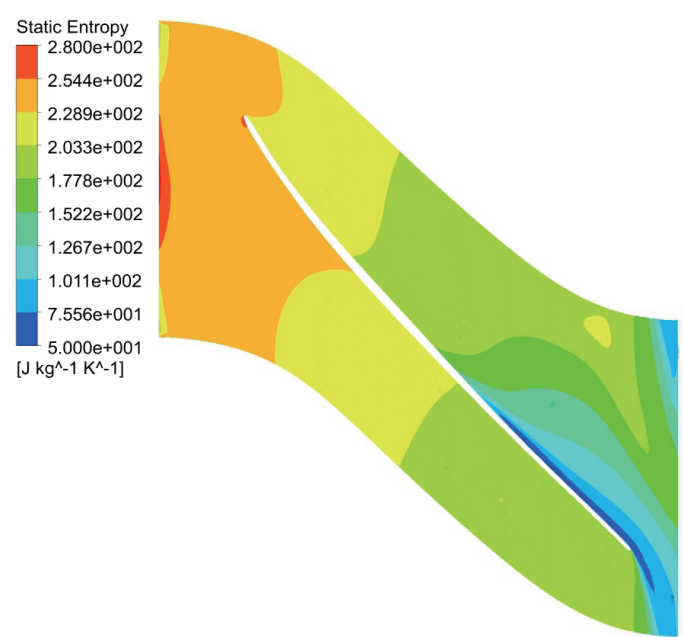

(c)

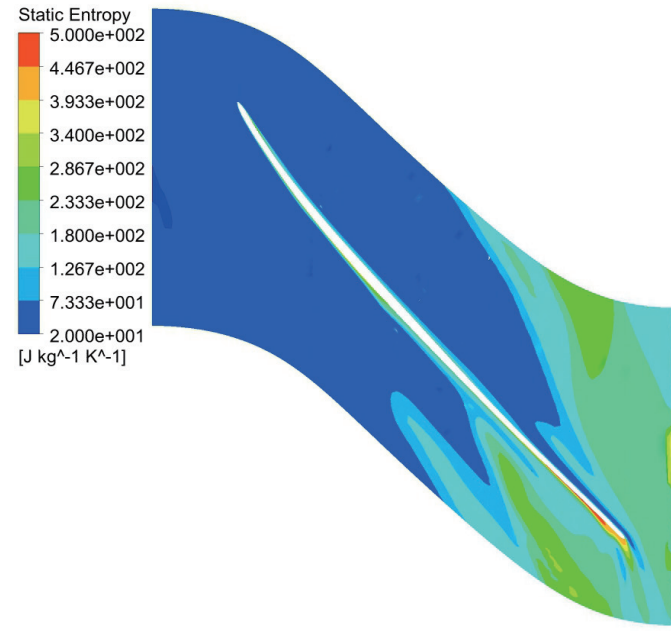

(b)



(d)

Fig. 11. (Color online) Static entropy of blade-to-blade section at blade height of 0.9. (a) Original blade at 0.0832 $\mathrm{kg} / \mathrm{s}$. (b) Original blade at $0.1781 \mathrm{~kg} / \mathrm{s}$. (c) New blade at $0.0832 \mathrm{~kg} / \mathrm{s}$. (d) New blade at $0.1781 \mathrm{~kg} / \mathrm{s}$.

The increase in static pressure due to air compression is one of the target performances of the compressor. The pressure distributions along the meridional passage when the mass flow rate is $0.0832 \mathrm{~kg} / \mathrm{s}$ are shown in Fig. 12. The original blade and new blade have similar pressure patterns. When the mass flow rate is $0.1781 \mathrm{~kg} / \mathrm{s}$, the internal pressure distribution is different from that at $0.0832 \mathrm{~kg} / \mathrm{s}$. There is a continuous low-pressure region at the tip of the new wheel in the first half of the flow passage. At the outlet, the high-pressure area of the new wheel is large and continuous, while there is a high-pressure cloud at the hub, and there is a small belt with high pressure in the original wheel. The high-pressure area of the new wheel is $70 \%$ larger than that of the original wheel. The relative static pressure of the original wheel is only 59129.2 Pa, whereas that of the new wheel is $63271.9 \mathrm{~Pa}, 7.00 \%$ higher than that of the original wheel. 


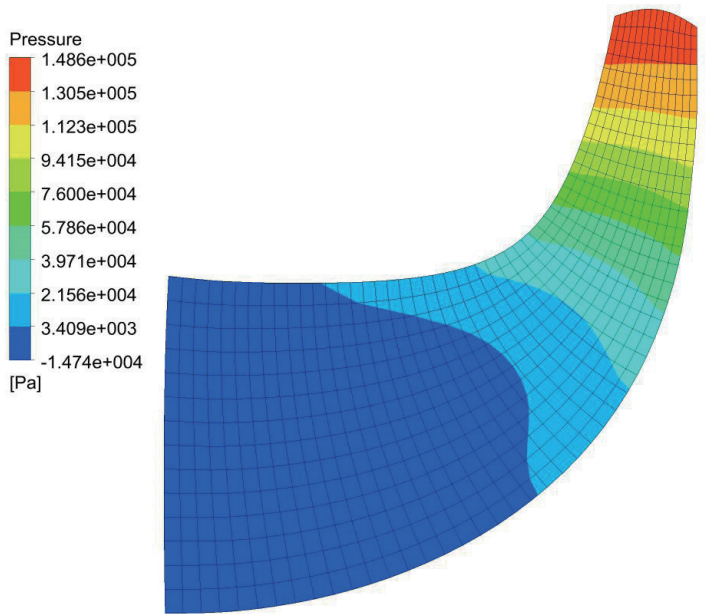

(a)

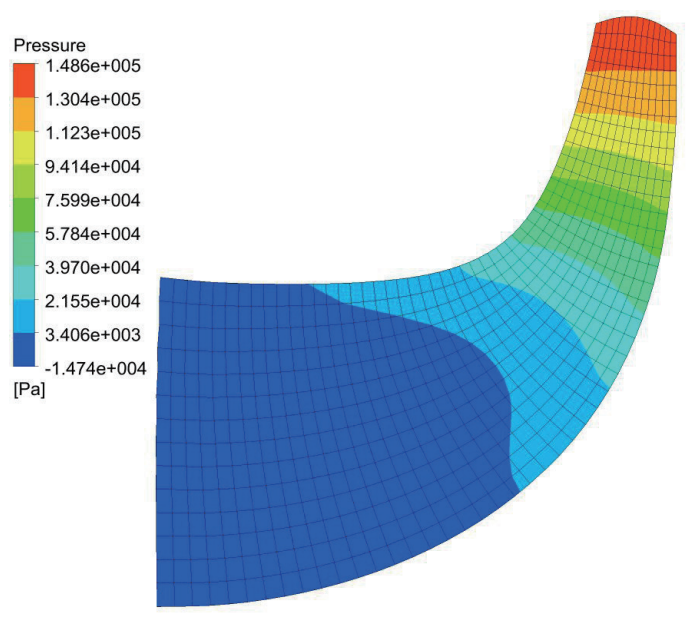

(c)

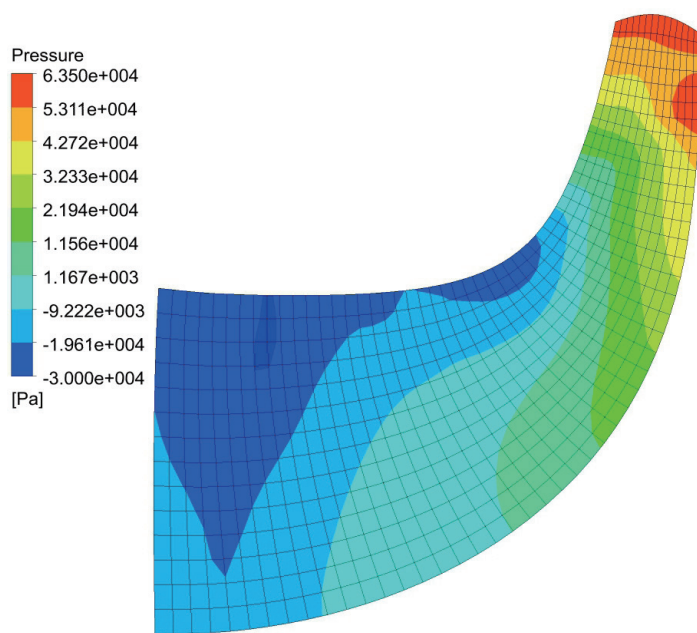

(b)

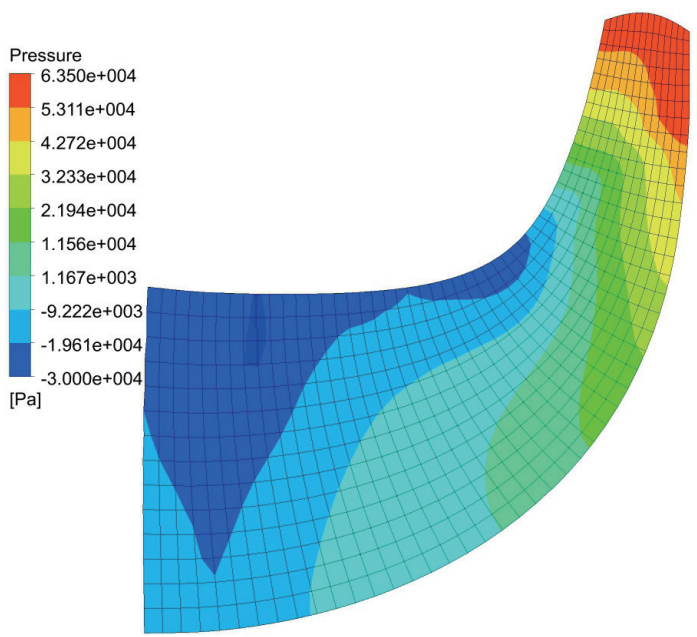

(d)

Fig. 12. (Color online) Static pressure distributions of meridional section. (a) Original blade at $0.0832 \mathrm{~kg} / \mathrm{s}$. (b) Original blade at $0.1781 \mathrm{~kg} / \mathrm{s}$. (c) New blade at $0.0832 \mathrm{~kg} / \mathrm{s}$. (d) New blade at $0.1781 \mathrm{~kg} / \mathrm{s}$.

During the operation of a compressor, both the air pressure and airflow velocity increase. The velocity profile distributions of a single passage at different mass flow rates are shown in Fig. 13. When the mass flow rate is $0.0832 \mathrm{~kg} / \mathrm{s}$, there is a strong secondary flow vortex line from the inlet to the outlet near the suction side, but it is relatively mild in the new wheel. There are also cyclone streamlines on the pressure side of the blade, which are gentler for the new blade. In the outlet region, the maximum velocity in the passage of the original compressor is $490.7 \mathrm{~m} / \mathrm{s}$, compared with $444.0 \mathrm{~m} / \mathrm{s}$ in the new compressor, a reduction of $9.51 \%$. When the mass flow rate is $0.1781 \mathrm{~kg} / \mathrm{s}$, the flow velocity profiles of the two passages are parallel and smooth along the blade contour direction. The maximum velocity at the trailing edge of the original compressor is $643.3 \mathrm{~m} / \mathrm{s}$, compared with $635.5 \mathrm{~m} / \mathrm{s}$ for the new compressor, a reduction of $1.21 \%$. 


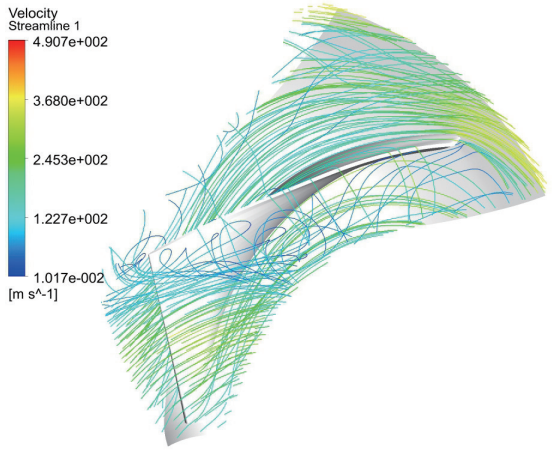

(a)

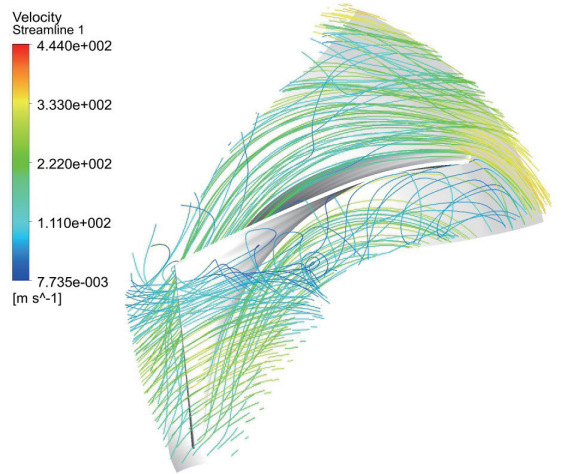

(c)

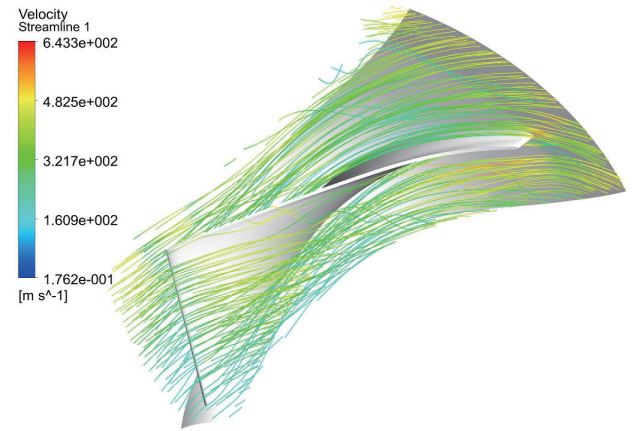

(b)

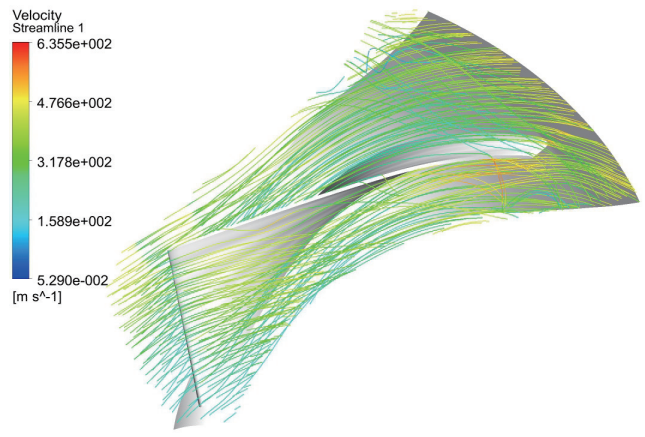

(d)

Fig. 13. (Color online) Velocity streamlines of a single passage. (a) Original blade at $0.0832 \mathrm{~kg} / \mathrm{s}$. (b) Original blade at $0.1781 \mathrm{~kg} / \mathrm{s}$. (c) New blade at $0.0832 \mathrm{~kg} / \mathrm{s}$. (d) New blade at $0.1781 \mathrm{~kg} / \mathrm{s}$

\section{Conclusions}

Considering both the mechanical properties and the compressor performance, a new blade with a bionic leading edge is designed using Bezier curves in BladeGen. With the topology grid number of 5,500,000 in TurboGrid, the $\mathrm{Y}+$ value of the rotating blade is less than 1, which ensures the generation of an appropriate grid and accurate analysis of the wheel blade.

The profile of the leading edge of the compressor blade is set as a ladder shape, and the step convexity height gradually increases from the hub to the shroud. The deformation of the new blade during rotation is less than that of the original blade, and the blade satisfies the mechanical requirements. In terms of the aerodynamic parameters, the new wheel has the advantages of increased pressure ratio and efficiency at low and high mass flow rates, but its efficiency decreases slightly by about $1 \%$ at intermediate mass flow rates with a turbulent flow.

The aerodynamic parameters show that the new blade causes little disturbance in the flow field. This ensures that the airflow is closer to the blade profile for a parallel flow and the velocity streamline cyclone phenomenon is weakened. The increase in entropy is also reduced, which is conducive to increasing the pressure ratio and efficiency. These are crucial elements beneficial for the blade material and performance, as well as providing good operation conditions for the sensors. 


\section{Acknowledgments}

This study and some of the analyses were supported by Fujian Province Key Laboratory of Ship and Ocean Engineering.

\section{References}

1 T. Korakianitis, A.Hamakhan, M. A. Rezaienia, A. P. S. Wheeler, E. J. Avital, and J. J. R. Williams: Appl. Energy 89 (2012) 215. https://doi.org/10.1016/j.apenergy.2011.07.004

2 S. Anish and N. Sitaram: J. Mech. Sci. Technol. 31 (2017) 5291. https://doi.org/10.1007/s12206-017-1023-2

3 J. M. Ni, H. He, Q. W. Wang, X. Y. Shi, and G. D. Li: Chin. Internal Combust. Engine. Eng. 35 (2014) 63. https://doi.org/10.13949/j.cnki.nrjgc.2014.01.014

4 Z. X. Yang, G. S. Li, L Song, and Y. F. Bai: 2017 Int. Conf. New Energy and Future Energy System, IOP Conference Series-Earth and Environmental Science (NEFES, 2017) 22-25. https://doi.org/10.1088/17551315/93/1/012041

5 A. Zamiri, B. J. Lee, and J. T. Chung: J. Mech. Sci. Technol. 31 (2017) 5557. https://doi.org/10.1007/s12206-017$1050-\mathrm{Z}$

6 M. Kaneko and H. Tsujita. J. Therm. Sci. 24 (2015) 117. https://doi.org/10.1007/s11630-015-0763-5

7 Z. M. Tong, J. L. Liu, J. M. Ni, Q. W. Wang, and W. Hou: Chin. Internal. Combust. Engine. Eng. 35 (2014) 107. https://doi.org/10.13949/j.cnki.nrjgc.2014.02.001

8 B. Mischo, P. Jenny, S. Mauri, Y. Bidaut, M. Kramer, and S. Spengler: J. Turbomach. 140 (2018) 111006. https:// doi.org/10.1115/1.4041400

9 S. M. Sun, C. Y. Xu, L. Q. Ren, and Y. Z. Zhang: J. Jilin Univ. (Eng. and Technol. Ed.) 39 (2009) 382. https:// doi.org/10.13229/j.cnki.jdxbgxb2009.02.009

10 L. Q. Ren, S. M. Sun, and C. Y. Xu: J. Jilin Univ. (Eng. and Technol. Ed.) 38 (2008) 126. https://doi. org/10.13229/j.cnki.jdxbgxb2008.s1.008

11 C. Y. Xu, Z. H. Qian, Q. P. Liu, S. M. Sun, and L. Q. Ren: J. Jilin Univ. (Eng. and Technol. Ed.) 40 (2010) 108. https://doi.org/10.13229/j.cnki.jdxbgxb2010.01.006

12 G. H. Liao, Q. C. Hu, Y. Yang, Z. W. Han, L. Q. Ren, and Q. P. Liu: J. Jilin Univ. (Eng. and Technol. Ed.) 42 (2012) 1163. https://doi.org/10.13229/j.cnki.jdxbgxb2012.05.029

13 B. Denkena, T. Grove, and T. Krawczy: 5th CIRP Global Web Conf. Research and Innovation for Future Production (Procedia CIRP) 55 (2016) 182. https://doi.org/10.1016/j.procir.2016.09.010

14 C. J. Ge, M. C. Ge, P. Liang, Z. H. Zhang, and L. Q. Ren: Trans. Chin. Soc. Agric. Mach. 44 (2013) 292. https:// doi.org/10.6041/j.issn.1000-1298.2013.S1.053

15 C. Xu, L. Chen, and R. S. Amano: J. Energy Res. Technol. 142 (2020) 081307. https://doi.org/10.1115/1.4046525 